\title{
Measurement of diabetes, prediabetes and their associated risk factors in Myanmar 2014
}

This article was published in the following Dove Press journal: Diabetes, Metabolic Syndrome and Obesity:Targets and Therapy

\author{
Tint-Swe Latt ${ }^{1,2}$ \\ Ko-Ko Zaw ${ }^{3}$ \\ $\mathrm{Ko} \mathrm{Ko}{ }^{4}$ \\ Moh-Moh Hlaing ${ }^{3}$ \\ Mya Ohnmar ${ }^{3}$ \\ Ei-Sandar $\mathrm{Oo}^{5}$ \\ Kyaw-Myint-Myat Thein ${ }^{6}$ \\ Motoyuki Yuasa ${ }^{2}$
}

'Myanmar Diabetes Association, Yangon, Myanmar; ${ }^{2}$ Department of Public Health, Juntendo University, Graduate School of Medicine, Tokyo, Japan; ${ }^{3}$ Department of Medical Research, Ministry of Health and Sports, Naypyidaw, Myanmar;

${ }^{4}$ University of Medicine 2, Yangon, Myanmar; ${ }^{5}$ University of Public Health, Yangon, Myanmar; ${ }^{6}$ Diabetes Project, Department of Health, Ministry of Health and Sports, Naypyidaw, Myanmar
Correspondence: Tint-Swe Latt Myanmar Diabetes Association, Yangon, Myanmar Email proftsı@gmail.com
Background: Myanmar, a low-income Southeast Asia country with an estimated population of 53.9 million, is currently facing an increasing burden of noncommunicable diseases. Research that systematically measures and reports the national prevalence of diabetes and prediabetes within Myanmar represents a gap in the literature. This study, a national survey, was conducted with the objectives of 1) measuring diabetic population, 2) measuring the prediabetic population, and 3) finding out the risk factors leading to diabetes and prediabetes in Myanmar.

Method: Multistage sampling was applied in proportional cluster approach to recruit a nationally representative sample of 8,575 participants from 52 townships selected to represent 332 townships across the country. The Ethical Committee of the Department of Medical Research (Lower Myanmar) approved the current study. Fasting plasma glucose and the level of glucose 2 hours after $75 \mathrm{~g}$ glucose load were applied, to screen for diabetes and prediabetes. Intervieweradministered questionnaires were carried out to investigate demographic and lifestyle behaviors. Stadiometer, weighing scale, measuring tape, and Omron blood pressure monitor measuring devices were used for the anthropometric measurements. Survey data analysis used STATA version 13 and application of descriptive statistics with multinomial logistic regression.

Result: Prevalence of diabetes in Myanmar was 10.8\%: $11.5 \%$ in males and $9.2 \%$ in females. Overall prevalence of prediabetes was $19.7 \%$ : $16.5 \%$ in males and $23 \%$ in females. Increasing age, urban residence, big waist circumference, and high triglyceride level were significant factors associated with diabetes and prediabetes. Many people with diabetes were found to lack knowledge and awareness of diabetes.

Conclusion: Diabetes prevalence in Myanmar is high, and relatively higher than that reported in many Association of Southeast Asian Nations countries. The study findings call for urgent nationwide public health action in Myanmar such as screening people at risk of diabetes and prevention of the disease. The findings can also assist the health authority in Myanmar with the preparation and allocation of resources for diabetes prevention.

Keywords: Yangon, waist circumference, prediabetes, diabetes, triglyceride

\section{Introduction}

Diabetes is a global issue. Some 425 million people worldwide, or $8.8 \%$ of adults 20-79 years, are estimated to have diabetes. About 79\% live in low and middle income countries. ${ }^{1}$ The percentage of deaths attributable to high blood glucose or diabetes that occurs prior to age 70 is higher in low- and middle-income countries than in highincome countries. ${ }^{2}$ With speedy urbanization and motorization in low-income countries with underdeveloped infrastructure, the risk factors associated with noncommunicable diseases (NCDs) such as type 2 diabetes, hypertension, and cardiovascular diseases 
(CVD) became higher. Estimates of the current and future burden of diabetes are essential in order to appropriately allocate resources, drive health-promoting policies, and encourage action to prevent diabetes in future generations. ${ }^{3}$

It is estimated that the number of adults with diabetes will increase by $55 \%$ by 2035 to 592 million. ${ }^{3}$ In addition to lifestyle changes trending toward obesity, unhealthy diets, and lack of physical activity, global population aging may also bring a higher prevalence of diabetes in many developing countries. ${ }^{1}$ The global prevalence of diabetes among people aged 20 and above was estimated to be $8.3 \%$ in 2013. ${ }^{3}$ The World Diabetes Federation estimated that the prevalence of diabetes for people aged 20 and above in Myanmar was $5.7 \%$ in 2013 and projects it to be $8 \%$ in $2035 .{ }^{3}$ However, recent reports within the Association of Southeast Asian Nations (ASEAN) community have indicated a faster rise in diabetes prevalence among Southeast Asian developing countries. ${ }^{4,5}$

Myanmar is a Southeast Asian developing country with a population of 53.9 million and the lowest life expectancy among the ASEAN countries. ${ }^{6,7}$ It is a low-income country that has recently undergone several transformations such as a shift to an electoral democratic political system, civil structural changes, sociodemographic changes, and economic transition. ${ }^{7,8}$ The global trend in the changing epidemiology of diseases has also been seen in Myanmar. Despite its out-of-the-pocket expenditure, hospitals in Myanmar have seen a growing number of chronic disease patients such as of diabetes, hypertension, stroke, and cancer, while infectious diseases are still forming a huge burden. ${ }^{6}$ The prevalence of diabetes has increasingly been reported in many local studies and clinical reports. ${ }^{9}$ However, evidence gathered from a nationally representative survey has still been lacking. The current study attempts to fill this research gap. ${ }^{10}$

The World Health Organization (WHO) has proposed a stepwise approach for the effective prevention and control of NCDs, with estimation of the problem magnitude as the first step (STEP). ${ }^{11}$ STEP surveys were conducted in Myanmar before 2014 through the collaboration of the Prevention and Control of Diabetes Project, and the Department of Medical Research, Myanmar. However, those were subnational surveys confined only to Yangon Division and unable to include comprehensive measurement such as blood glucose level and blood cholesterol level assessment. ${ }^{12}$ The Myanmar STEP survey in 2014 was the first time a national survey had been carried out, and one that amounted to a comprehensive measurement. The current study analyzed and reported the diabetes-focused data from this national STEP survey, particularly in relation to the prevalence of diabetes and prediabetes, and their associated risk factors.

The objectives were 1) to determine the national prevalence of type 2 diabetes mellitus in the adults in Myanmar, 2) to determine the national prevalence of prediabetes in the adults in Myanmar, and 3) to identify the risk factors associated with diabetes mellitus and prediabetes. Ultimately, this study aimed to contribute to the policy development, agenda setting and strategic action plan for prevention of diabetes and prediabetes and their associated risk factors.

\section{Methodology Study design}

The household-based cross-sectional design, based on the methodology of the WHO STEPwise approach to surveillance of major NCD risk factors, was utilized. The core and expanded indicators of Steps 1, 2, and 3 were included. The study population consisted of people aged from 25 to 64 years of both sexes residing in both urban and rural areas of the country. The study excluded the following people: mentally ill people, seriously ill people, institutionalized individuals (members of the armed forces, hospitalized patients, prisoners), and temporary residents (those living in a locality for less than 6 months).

\section{Sample size}

The sample size was calculated using the following formula:

$$
N=Z_{z}^{2} \times P(1-P) / e^{2}
$$

where $N$ is the required sample size, $Z_{z}$ the $Z$ statistics for predetermined alpha error, $P$ the estimated prevalence of diabetes mellitus, and $e$ is the margin of error.

In the present study, the value of 1.96 was taken as $Z_{z}$ for $95 \%$ confidence and the estimated prevalence of diabetes mellitus was taken as $4.6 \%$, referring to the global prevalence of diabetes mellitus in $2000 .^{13,14}$ The margin of error was taken as $5 \%$. The required sample size, therefore, was 160 . As this study intended to calculate diabetes prevalence rates in eight sex-age groups and four geographical regions (hilly, coastal, central plain, and delta regions) the sample size needed to be adjusted by a factor of 1.5 for cluster sampling and a factor of $(1 / 0.85)$ for a $15 \%$ nonresponse rate. The final required sample size for the whole study, therefore, turned out to be $9,036[160 \times 8 \times 4 \times(1 / 0.85)]$, which was rounded to 9,360 for logistical reasons. 


\section{Sampling approach}

Multistage cluster sampling was used to recruit the study subjects. In step 1, a total of 52 townships were chosen from the 330 townships of Myanmar. Probability proportionate to size method was applied. In step 2, 6 wards and villages were selected from each of the 52 selected townships. In step 3, 30 households were selected from each selected ward/ village using systematic sampling. One eligible participant responded survey, from each chosen household using the Kish table method. A total of 8,757 participants formed the representative sample for the national survey. In Myanmar, each region has townships accommodating more city-like and villages accommodating more countryside communities. We considered people from townships as urban and people from villages as rural. Sampling was carefully designed to recruit urban and rural residents proportionately in each region.

\section{Sampling weights}

Sampling weights (the number of the population represented by a respondent) were given by age and sex as in Table 1 .

\section{Data collection}

The Ethical Committee of the Department of Medical Research (Lower Myanmar) approved the current study to conduct verbal informed consent of the participants. The duration of data collection was from September to December, 2014. Participants provided verbal consents after receiving explanation about the study. The trained interviewers collected data relating to sociodemography, health behaviors, anthropometry, and blood pressures (BPs) via face-to-face interviews and physical examinations. Ten-hour overnight fast was requested in advance. Fasting capillary blood samples were tested for plasma glucose (PG) and lipids, and capillary blood samples were tested for glucose 2 hours after glucose load. BP measure was preceded by a resting time of 10 minutes before measurement.

In addition to the questionnaire instrument, survey tools for measurement consisted of a stadiometer for measuring height, a weighing scale, measuring tape for waist and hip measurement,

Table I Sampling weights (number of people represented by a respondent) by age and sex

\begin{tabular}{|l|l|l|l|}
\hline Age group (years) & Men & Women & Both sexes \\
\hline $25-34$ & 6,230 & 3,520 & 4,502 \\
\hline $35-44$ & 4,004 & $\mathrm{I}, 950$ & 2,624 \\
\hline $45-54$ & 2,864 & $\mathrm{I}, 573$ & 2,030 \\
\hline $55-64$ & 2,489 & $\mathrm{I}, 45 \mathrm{I}$ & $\mathrm{I}, 832$ \\
\hline $25-64$ & 3,785 & 2,049 & 2,660 \\
\hline
\end{tabular}

and an Omron BP monitor. The lipid profile, fasting plasma glucose (FPG), and 2 hours PG were determined on-site using a portable LipidoCare device. Preliminary data were allowed to republish by authors of presentation, Latt TS et al, and with the permission of the president of Myanmar Diabetes Association..$^{15}$

\section{Data analysis}

Diabetes mellitus is defined as having an FPG concentration of $>7.0 \mathrm{mmol} / \mathrm{L}$ and/or a $\mathrm{PG}$ concentration of $>11.1$ $\mathrm{mmol} / \mathrm{L} 2$ hours after $75 \mathrm{~g}$ oral glucose load or the taking of current medication for diabetes. Prediabetes refers to IGT and impaired fasting glucose (IFG). IGT refers to 2-hour PG level of $140 \mathrm{mg} / \mathrm{dL}(7.8 \mathrm{mmol} / \mathrm{L})$ but $<200 \mathrm{mg} / \mathrm{dL}(11.1$ $\mathrm{mmol} / \mathrm{L})$. IFG refers to an FPG level of $110 \mathrm{mg} / \mathrm{dL}(6.1$ $\mathrm{mmol} / \mathrm{L})$ but $<126 \mathrm{mg} / \mathrm{dL}(7.0 \mathrm{mmol} / \mathrm{L})$. (5) Definitions are as follows:

1. Central obesity in terms of abnormal waist: waist circumference $\geq 90 \mathrm{~cm}$ for males and $\geq 80 \mathrm{~cm}$ for females were defined as abnormal waist circumference. ${ }^{17,18}$

2. High triglycerides level: triglycerides $\geq 150 \mathrm{mg} / \mathrm{dL}$ $(\geq 1.7 \mathrm{mmol} / \mathrm{L})$, or the taking of treatment for this lipid abnormality ${ }^{16}$ :

3. Reduced high-density lipoprotein (HDL)-cholesterol: HDL-cholesterol $<40 \mathrm{mg} / \mathrm{dL}(<1.03 \mathrm{mmol} / \mathrm{L})$ for males and $<50 \mathrm{mg} / \mathrm{dL}(<1.29 \mathrm{mmol} / \mathrm{L})$ for females, or the taking of treatment for this lipid abnormality.

4. High BP: systolic $\mathrm{BP} \geq 130$ or diastolic $\mathrm{BP} \geq 85 \mathrm{mmHg}$, or treatment of previously diagnosed hypertension. ${ }^{17,18}$

Complex sampling designs (stratified sampling, cluster sampling, and unequal sampling weights) were controlled at every step of data analysis using "svy" (survey) commands in the statistical software Stata Version 13.0. The prevalence of diabetes and prediabetes was presented as percentages with a $95 \%$ CI for both sexes and four age groups.

Multinomial logistic regression was carried out to determine the independent effect of each of the risk factors on diabetes and prediabetes. Selection of the variables for inclusion as well as the modeling procedure was done according to the guidelines prescribed by David W. Hosmer and Stanley Lemeshow. ${ }^{6}$

Initially, 13 variables (age, sex, urban-rural residence, household annual income, smoking rate, intake of alcohol, intake of fruit and vegetables, physical activity, central obesity, hypertension, total blood cholesterol, fasting triglycerides, and HDL) were included and modeled, using backward selection and a 0.05 significance level. In the final model, only five variables (sex, age, urban-rural residence, central obesity, and high triglycerides) remained: the other eight 
variables were omitted due to their statistical insignificance and minor contribution to the model.

\section{Results}

Out of the 9,360 respondents scheduled to participate in the study, a total of 8,757 respondents (93.6\%) completed all of the required assessments: questionnaire, physical measurement, and blood glucose level measurement.

\section{Background characteristics}

Female respondents made up $65 \%$ of the study population. The age group of 45-54 years formed the highest proportion in both sexes. Eighty-four percent of the men and $71 \%$ of the women in the study group were married.

\section{Prevalence of diabetes}

Table 2 presents the percentage of the respondents with diabetes (both previously diagnosed and previously undiagnosed but detected in the current survey). Overall, the prevalence of diabetes was $10.5 \%$, with $9.1 \%$ in the men and $11.8 \%$ in the women. Among the men, the prevalence of diabetes increased as age increased from $3.6 \%$ in the $25-34$-year age group to $19.5 \%$ in the 55-64-year age group. Among the women the prevalence of diabetes also increased as age increased from $4.5 \%$ in the $25-34$ year age group to $23.7 \%$ in the 55-64-year age group. Seven out of ten diabetic respondents were previously undiagnosed.

\section{Regional variation in the prevalence of diabetes}

Figure 1 shows the prevalence of diabetes and prediabetes in 14 different states and regions of Myanmar. The overall prevalence of diabetes was the highest in Yangon region $(18.2 \%)$ and the lowest in Nayphitaw (4.2\%). In all states and regions, with the exception of Yangon, $<30 \%$ of the people were aware of their own diagnosis. All states and regions, with the exception of Yangon, had a higher percentage of prediabetes than of diabetes.

\section{Urban/rural difference in prevalence of diabetes according to the area of residence}

The prevalence of diabetes in the urban population $(14.4 \%$, $95 \% \mathrm{CI}=12-13$ ) was higher than that in the rural population (9\%, 95\% CI $=6.8-11.8)$.

\section{Types of glucose tolerance}

Figure 2 shows the distribution of different types of glucose tolerance. IGT and FGT (IGT alone, FGT alone, and the combined presence of IGT and FGT) make up prediabetes, of which the prevalence is $20 \%$. The prevalence of IGT alone $(17 \%)$ is much higher than that of FGT alone $(2 \%)$ and of combined IGT and FGT (1\%).

\section{Associated factors of diabetes and prediabetes}

Five factors were significant and retained in the final model of backward stepwise multivariate analysis measuring the association of independent variables to prediabetes. Those included three demographic factors: female gender, older age groups, urban residence, and two biologic factors: central obesity and higher fasting triglycerides. Those who have a bigger waist circumference are more likely to have predia-

Table 2 Prevalence of diabetes in Myanmar, 2014

\begin{tabular}{|c|c|c|c|c|c|c|c|c|}
\hline \multirow[t]{2}{*}{ Sex } & \multirow{2}{*}{$\begin{array}{l}\text { Age in } \\
\text { years }\end{array}$} & \multirow[t]{2}{*}{ Population } & \multicolumn{2}{|c|}{ Diagnosed diabetes } & \multicolumn{2}{|c|}{ Undiagnosed diabetes } & \multicolumn{2}{|c|}{ Total diabetes } \\
\hline & & & $\%$ & $95 \% \mathrm{Cl}$ & $\%$ & $95 \% \mathrm{Cl}$ & $\%$ & $95 \% \mathrm{Cl}$ \\
\hline \multirow[t]{5}{*}{ Men } & $25-34$ & 616 & 0.1 & $0.0,0.4$ & 3.5 & $1.5,8.0$ & 3.6 & $1.6,8.1$ \\
\hline & $35-44$ & 738 & 1.7 & $0.9,3.1$ & 5.9 & $3.7,9.2$ & 7.6 & $5.0,11.2$ \\
\hline & $45-54$ & 832 & 4.8 & $2.5,8.9$ & 7.0 & $5.2,9.4$ & 11.8 & $8.7,15.9$ \\
\hline & $55-64$ & 707 & 3.5 & $2.0,6.0$ & 16.0 & $10.4,23.6$ & 19.5 & $13.4,27.4$ \\
\hline & $25-64$ & 2,893 & 2.1 & $1.4,3.1$ & 7.0 & $5.3,9.1$ & 9.1 & $6.9,11.8$ \\
\hline \multirow[t]{5}{*}{ Women } & $25-34$ & $\mathrm{I}, 099$ & 0.4 & $0.2,1.0$ & 4.1 & $2.7,6.2$ & 4.5 & $3.1,6.6$ \\
\hline & $35-44$ & $\mathrm{I}, 559$ & 2.3 & $1.4,3.7$ & 8.7 & $5.6,13.3$ & 11.0 & $7.9,15.0$ \\
\hline & $45-54$ & $\mathrm{I}, 537$ & 5.3 & $3.6,7.7$ & 10.3 & $8.1,13.1$ & 15.6 & $12.6,19.1$ \\
\hline & $55-64$ & $\mathrm{I}, 236$ & 10.7 & $7.5,15.1$ & 13.0 & $8.6,19.2$ & 23.7 & $19.2,28.8$ \\
\hline & $25-64$ & $5,43 I$ & 3.7 & $2.6,5.1$ & 8.2 & $6.0, I I . I$ & 11.8 & $9.6,14.6$ \\
\hline \multirow[t]{5}{*}{ Both sexes } & $25-34$ & 1,715 & 0.2 & $0.1,0.5$ & 3.8 & $2.4,6.0$ & 4.1 & $2.6,6.2$ \\
\hline & $35-44$ & 2,297 & 2.0 & $1.3,3.0$ & 7.3 & $5.0,10.4$ & 9.3 & $6.8,12.4$ \\
\hline & $45-54$ & 2,369 & 5.0 & $3.6,7.0$ & 8.7 & $7.2,10.4$ & 13.7 & $11.3,16.6$ \\
\hline & $55-64$ & $\mathrm{I}, 943$ & 7.1 & $5.0,10.1$ & 14.5 & $9.8,20.9$ & 21.6 & $16.5,27.7$ \\
\hline & Total & 8,324 & 2.9 & $2.2,3.8$ & 7.6 & $5.7,9.9$ & 10.5 & $8.3,13.1$ \\
\hline
\end{tabular}




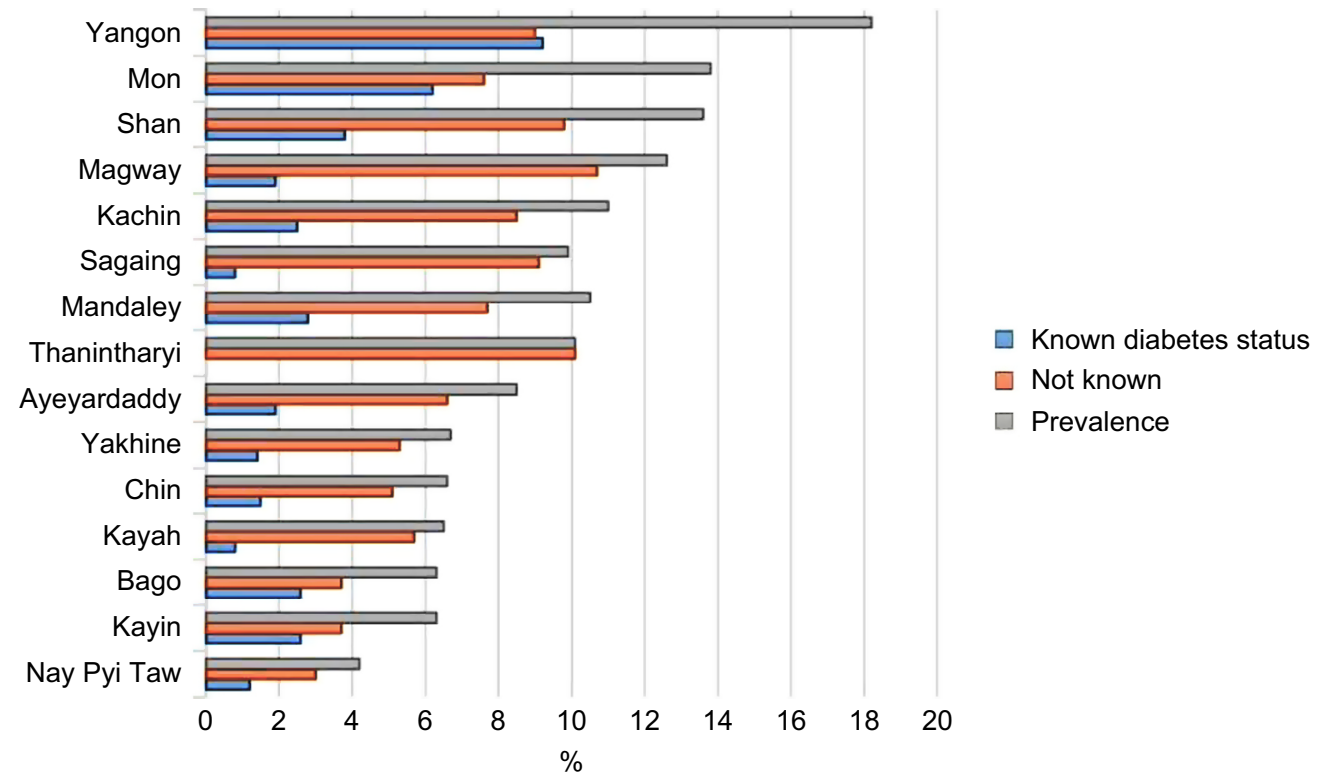

Figure I Prevalence of diabetes by state and region, Myanmar, 2014.

Notes: In each region urban and rural population was equally sampled.

Abbreviation: MMDA, Myanmar Diabetes Association.

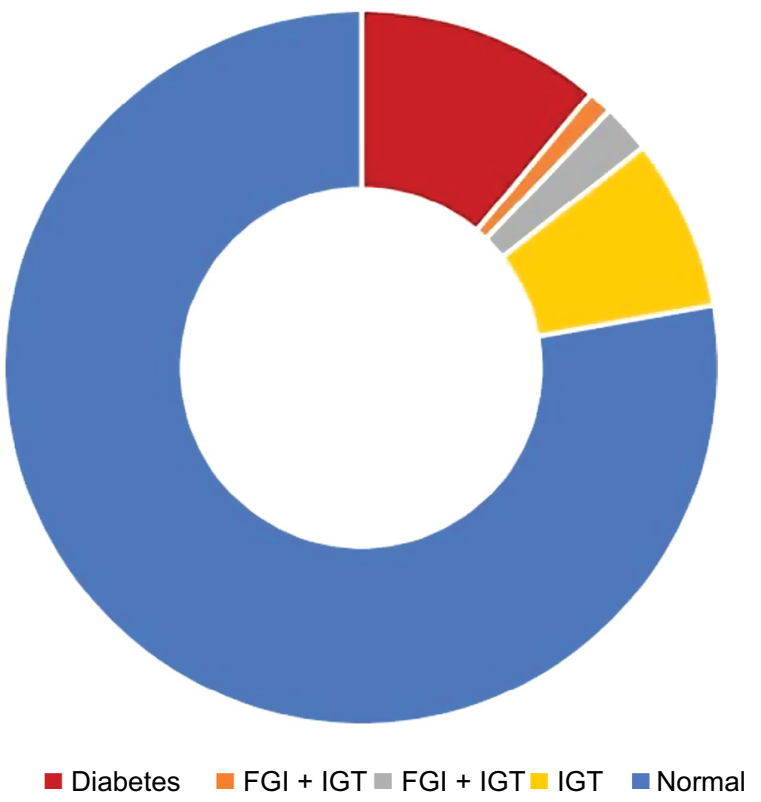

Figure 2 Types of glucose tolerance, Myanmar, 2014.

Abbreviations: IGT, impaired glucose tolerance; FGl, fasting glucose intolerance; MMDA, Myanmar Diabetes Association.

betes and diabetes (relative risk ratio [RRR] for diabetes 1.4 [1.1-1.8], RRR for prediabetes 3.1 [2.4-4.2]) and those with high triglyceride level (RRR for diabetes 1.3 [1.0-1.6], RRR for prediabetes 1.7 [1.3-2.2]).

Females are more likely to have prediabetes and diabetes than males (RRR for diabetes 1.5 [1.2\%-1.8\%], RRR for prediabetes 1.3 [1.0-1.8]). The risk of diabetes persistently increases in older age groups compared to the 25-34-year age group: RRR for diabetes 1.7 (1.3-2.2), RRR for prediabetes 2.5 (1.6-4.0) in 35-45-year age group, RRR for diabetes 2.0 (2.0-3.8), RRR for prediabetes $3.4(2.1-5.3)$ in 45-54-year age group, and RRR for diabetes 2.7 (2.0-3.8), RRR for prediabetes 6.4 (3.9-10.3) in 55-64-year age group. People living in more urbanized areas are more likely to have prediabetes and diabetes (RRR for diabetes 1.1 [0.8-1.3], RRR for prediabetes $1.5[1.3-2.2])$.

\section{Discussion}

This study is a comprehensive national survey investigating prevalence of diabetes and prediabetes in Myanmar. It is the first of its kind to provide robust evidence of the national burden of diabetes. This evidence is important for launching a preventive strategy and as a contribution to reducing the national and global burden of diabetes. The high level of the response rate $(91 \%)$ and multistage cluster sampling resulted in the representativeness of the data and the generalizability of the results to the Myanmar population. Given the high prevalence of diabetes and prediabetes in Myanmar, there is a need for a nationwide diabetes screening intervention in all regions of Myanmar after the age of 35 years. Moreover, the risk factors shown to be associated with diabetes status highlight who should be focused on in the launching of a strategy to prevent diabetes and prediabetes. 
The national prevalence of diabetes in Myanmar in 2014 was $10.5 \%$, while prediabetes was nearly 2 times that of diabetes (19.7\%) (Figure 2). There is the possibility of prediabetes in every three out of ten adults in Myanmar. This represents a critical situation, which, without preventive intervention, may lead to a rapid, unprecedented rise in the prevalence of diabetes and its complications in the coming years. The higher prevalence of diabetes in Myanmar may be due to the different methodology for detection of diabetes. In this study, $75 \mathrm{~g}$ oral glucose tolerance test was applied, and OGTT usually give higher detection rate of diabetes than the fasting plasma glucose alone., ${ }^{3,5}$

Furthermore, many of the diabetic people may be unaware of their diabetic status due to the lack of an active screening program. The high rate of undiagnosed diabetes and prediabetes calls for urgent action that can provide opportunities for communities to screen blood glucose levels and risk factors associated with diabetes. Furthermore, investment in future research that investigates public awareness and health literacy of diabetes in Myanmar is required. ${ }^{19}$

In a recent study, Latt et $\mathrm{al}^{20}$ reported the gap and challenge of integrating health services for diabetes in Myanmar. Research findings on the prevalence and risk factors for diabetes are necessary in order to put into place an effective national policy of screening and early lifestyle intervention of the disease. The current study has sought to fill this need. The result of multinomial logistic regression in the present study indicates that older age groups, urban residence central obesity, and higher fasting triglycerides represent significant risk factors contributing to diabetes and prediabetes within Myanmar's adult population.

The findings showed that there is an increasing risk of diabetes and prediabetes in older age groups. The risk for prediabetes and type 2 diabetes became higher in the older age group. This highlights the factor of population aging as the driver of diabetes epidemics. ${ }^{21}$ Moreover, because of comorbidities and polypharmacy, diabetes can have a profound impact on the daily life activities of elderly people resulting in disability and deaths. ${ }^{21} \mathrm{~A}$ screening program that is able to detect diabetes among people in high-risk age groups should be introduced as a public health intervention in order to preserve the quality of life of the elderly.

Developing countries such as Myanmar are undergoing rapid urbanization and lifestyle changes. In recent years, cities in Myanmar have seen rapid motorization and increased sedentary lifestyles amongst urban dwellers. ${ }^{7,22}$ Moreover, urban residents rely more on readily available food from shops and fast food stalls that have limited healthy choices. Moreover, facilities and an environment for exercise and physical activities may be of limited access. Where there is a lack of infrastructure and opportunity to sustain an active lifestyle, citizens usually have a low level of physical activity, which may lead to diabetes. ${ }^{23,24}$ These factors may explain the prevalence of diabetes among urban residents in Myanmar.

Central obesity, defined by measuring waist and hip circumference, represents a significant risk factor for diabetes. Research findings within the Asian setting indicate waist circumference as a potentially sensitive tool for detecting prediabetes and metabolic obesity. ${ }^{25}$ The typical morphology of a small body with a fat belly and big waistline is known to be an indicator of metabolic obesity among Asians, which is a predictor of metabolic diseases. ${ }^{26}$ Therefore, the current study findings are consistent with such research findings within the Asia setting. This basic finding has the potential to be practically applied as a screening tool. Waist circumference measurement can be used to screen for diabetes and prediabetes in the community setting in Myanmar. Identifying at-risk people and recommending lifestyle modifications can help to prevent diabetes and avert health expenditure for the lifelong treatment of diabetes and its complications.

The current study has identified hypertriglyceridemia as a significant risk factor for diabetes and prediabetes within the population of Myanmar. This is consistent with the finding from a cohort study carried out in the UK of more than 4,000 people over a 12-year period, which reported the association of a high triglyceride level and the risk of type 2 diabetes. ${ }^{27}$ Moreover, this finding reflects the risk of diabetes among the people of Myanmar because of the traditional cooking style, which is oily and involves the consumption of the highcarbohydrate staple food, rice. Research leading to dietary intervention and health education is important in order to promote the public's knowledge of a healthy diet. Health education, which incorporates practical cooking lessons, would be beneficial to the prevention of diabetes.

The silent epidemic of diabetes is widespread in Myanmar. Diabetes may bring many other NCDs such as CVD, strokes, disability, and deaths as its complications, resulting in heavy health care costs for this low-income country. Simple lifestyle interventions such as promoting a healthy diet and physical activity are effective in the prevention of diabetes. However, the way to integrate these within the country's social and cultural context is challenging. The findings of the current study may serve to contribute to the design of an effective health promotion intervention for the prevention of diabetes in Myanmar.

\section{Strengths and limitations}

The strength of this study is its generalizability due to its nationally representative sample and good response rate. IGT 
was used to screen individuals for prediabetes and diabetes, enhancing its internal validity. The study was not without limitations. According to the details of the STEP survey, people who were on antidiabetic medication or admitted to the hospital at the time of survey were not included in the survey. This might cause a slight underestimation of the prevalence of diabetes.

\section{Conclusion}

This study contributes updated evidence for increasing prevalence of known and unknown diabetes mellitus and prediabetes in Myanmar. Diabetes prevalence in Myanmar is high, and relatively higher than other ASEAN countries. To screen at-risk individuals and provide early lifestylerelated intervention, evidence-based risk factors of diabetes and prediabetes are necessary in each country. The findings in this study, therefore, may contribute to the integration of screening and prevention of diabetes within primary health care-based services of Myanmar and similar socioculturally developing countries in Southeast Asia.

\section{Acknowledgments}

The World Diabetes Foundation is acknowledged for its financial support for the study. WHO SEAR is acknowledged for its technical support for the study. Dr Myo Nyein Aung, Juntendo University, is acknowledged for his co-ordination and advice for writing up of the article.

\section{Disclosure}

The authors report no conflicts of interest in this work.

\section{References}

1. International Diabetes Federation, IDF Diabetes Atlas, Eighth Edition; 2017. Available from: http://diabetesatlas.org/resources/2017-atlas.html. Accessed June 31, 2013.

2. World Health Organization, Global Report on Diabetes. 2016. Available from: http://apps.who.int/iris/bitstream/handle/10665/204871/9789241565257_ eng.pdf;jsessionid $=5$ C33089E8CFDDB0F07CD792F4AE8FCD8?seque nce $=1$. Accessed December 31, 2016.

3. Guariguata L, Whiting D, Hambleton I, Beagley J, Linnenkamp U, Shaw J. Global estimates of diabetes prevalence for 2013 and projections for 2035. Diabetes research and clinical practice. 2014;103(2):137-149.

4. Picazo OF. Engendering Concerted National Efforts towards Improved Health Outcomes in the ASEAN: Status, Challenges, Targets, and Ways Forward. 2015. Available from: http://www.eria.org/ERIA-DP-2015-83. pdf. Accessed December 22, 2013.

5. Aekplakorn W, Abbott-Klafter J, Premgamone A, et al. Prevalence and Management of Diabetes and Associated Risk Factors by Regions of Thailand Third National Health Examination Survey 2004. Diabetes care. 2007;30(8).

6. Adelman MW, Kurbatova E, Wang YF, et al. Cost analysis of a nucleic acid amplification test in the diagnosis of pulmonary tuberculosis at an urban hospital with a high prevalence of TB/HIV. PloS one. 2014;9(7):e100649.
7. Codsi E, Tweet MS, Rose CH, Arendt KW, Best PJ, Hayes SN Spontaneous Coronary Artery Dissection in Pregnancy: What Every Obstetrician Should Know. Obstetrics and gynecology. 2016;128(4): 731-738.

8. Sanchez J. Understanding Reform in Myanmar: People and Society in the Wake of Military Rule Marie Lall. Hurst and Co Ltd, London, 2016, pp. xviii+ 346 (ISBN 9781849045803). Wiley Online Library; 2016.

9. Hlaing HH, Liabsuetrakul T. Dietary intake, food pattern, and abnormal blood glucose status of middle-aged adults: a cross-sectional community-based study in Myanmar. Food \& nutrition research. 2016;60.

10. World Health O. A Prioritized research agenda for prevention and control of noncommunicable diseases. Geneva :: World Health Organization; 2011.

11. World Health Organization. Preventing Chronic Diseases - A Vital Investment. Geneva, WHO, . 2005; http://www.who.int/chp/chronic_ disease_report/en/. Accessed 17.12.2016.

12. Latt TS, Min T, Aung PP. WHO STEPwise Approach to NCD Surveillance. Yangon, Myanmar: Ministry of Health. 2004.

13. Hosmer DW, Lemeshow S. Introduction to the Logistic Regression Model. Applied Logistic Regression: New York: John Wiley \& Sons, Inc.; 2005:1-30.

14. Hosmer DW, Lemeshow S. Multiple Logistic Regression. Applied Logistic Regression: John Wiley \& Sons, Inc.; 2005:31-46.

15. Myanmae Diabetes Association; Home page 2017; http://www.myanmardiabetes.org.mm/. Accessed 7.11.2017.

16. Alberti K, Eckel RH, Grundy SM, et al. Harmonizing the metabolic syndrome a joint interim statement of the international diabetes federation task force on epidemiology and prevention; national heart, lung, and blood institute; American heart association; world heart federation; international atherosclerosis society; and international association for the study of obesity. Circulation. 2009;120(16):1640-1645.

17. Janiszewski PM, Janssen I, Ross R. Does waist circumference predict diabetes and cardiovascular disease beyond commonly evaluated cardiometabolic risk factors? Diabetes Care. 2007;30(12):3105-3109.

18. Low S, Chin MC, Ma S, Heng D, Deurenberg-Yap M. Rationale for redefining obesity in Asians. Annals of the Academy of Medicine, Singapore. 2009;38(1):66-69.

19. Lorga T, Srithong K, Manokulanan P, Aung T, Aung MN. Public knowledge of diabetes in Karen Ethnic rural residents: a community-based questionnaires study in the far north-west of Thailand. Int J Gen Med. 2012;5:799-804.

20. Latt TS, Aye TT, Ko K, Zaw KK. Gaps and challenges to integrating diabetes care in Myanmar. 2016.

21. Kirkman MS, Briscoe VJ, Clark N, et al. Diabetes in Older Adults. Diabetes Care. 2012;35(12):2650-2664.

22. Dantas-Duarte A, Morais-de-Jesus M, Nunes AP, et al. Risk-taking behavior and impulsivity among HCV-infected patients. Psychiatry research. 2016;243:75-80.

23. Reis RS, Hino AAF, Rech CR, Kerr J, Hallal PC. Walkability and Physical Activity: Findings from Curitiba, Brazil. American journal of preventive medicine. 2013;45(3):269-275.

24. Association APH. Promoting active transportation: an opportunity for public health. Washington: American Public Health Association. 2012.

25. Lorga T, Aung MN, Naunboonruang P, et al. Predicting prediabetes in a rural community: a survey among the Karen ethnic community, Thasongyang, Thailand. International Journal of General Medicine. 2012;5:219-225.

26. Chan JC, Malik V, Jia W, et al. Diabetes in Asia: epidemiology, risk factors, and pathophysiology. JAMA. 2009;301(20):2129-2140.

27. Beshara A, Cohen E, Goldberg E, Lilos P, Garty M, Krause I. Triglyceride levels and risk of type 2 diabetes mellitus: a longitudinal large study. J Investig Med. 2016;64(2):383-387. 
Diabetes, Metabolic Syndrome and Obesity: Targets and Therapy is an international, peer-reviewed open-access journal committed to the rapid publication of the latest laboratory and clinical findings in the fields of diabetes, metabolic syndrome and obesity research. Original research, review, case reports, hypothesis formation, expert opinion and commentaries are all considered for publication. The manuscript management system is completely online and includes a very quick and fair peer-review system, which is all easy to use. Visit http://www.dovepress.com/testimonials.php to read real quotes from published authors.

Submit your manuscript here: https://www.dovepress.com/diabetes-metabolic-syndrome-and-obesity-targets-and-therapy-journal 